\title{
DECOMPOSIÇÃO E LIBERAÇÃO DE NITROGÊNIO DE RESÍDUOS CULTURAIS DE PLANTAS DE COBERTURA DE SOLO SOLTEIRAS E CONSORCIADAS ${ }^{(\mathbf{1})}$
}

\author{
C. $\operatorname{AITA}^{(2)} \&$ S. J . GIACOMINI(3)
}

\begin{abstract}
RESUMO
A dinâmica de decomposição e liberação de nitrogênio (N) de resíduos culturais provenientes de plantas de cobertura de solo, soltei ras e consorciadas, foi avaliada em um experi mento realizado em condições de campo, utilizando-se os seguintes materiais: (a) aveia preta (Avena strigosa Schieb); (b) ervilhaca comum (Vicia sativa L.); (c) nabo forrageiro (Raphanus sativus L. var. oleiferus Metzg.); (d) $32 \%$ de aveia preta $+68 \%$ de ervilhaca comum, e (e) $51 \%$ de aveia preta $+\mathbf{4 9} \%$ de ervil haca comum. Para monitorar o processo de decomposição, o material vegetal, seco ao ar, foi colocado em bolsas de nylon de 0,2 x 0,2 m e com mal ha de 0,5 mm, as quais foram deixadas na superfície do solo e recolhidas após 15, 29, 43, 59, 71, 82, 112 e 182 dias. Nos materiais coletados, foram feitas determinações das quantidades remanescentes de matéria seca (MS) e da sua concentração de N. Aos valores obtidos para as quantidades remanescentes de matéria seca e N, foram ajustados modelos não-lineares, com vistas em separar a MS e o $\mathrm{N}$ dos resíduos culturais em dois compartimentos, sendo um mais facilmente decomponível e outro mais recalcitrante. Dentre as espécies solteiras, a ervil haca foi a que apresentou a maior taxa de decomposição $\left(0,06119 \mathrm{dia}^{-1}\right) \mathrm{da}$ MS do compartimento mais facilmente decomponível. A presença da aveia nos consórcios com ervilhaca reduziu em $30 \%\left(0,04264 \mathrm{dia}^{-1}\right)$ e $50 \%\left(0,03113 \mathrm{dia}^{-1}\right)$ a decomposi ção da MS desse comparti mento, quando a proporção de matéria seca da gramínea foi de 32 e $51 \%$, respectivamente. Da quantidade total de $\mathrm{N}$ li berada durante os 182 dias do experimento, a maior parte ocorreu nos primei ros 15 dias. Os resultados deste estudo evidenciaram que, consorciando aveia e ervilhaca, não houve redução na taxa de liberação do $\mathbf{N}$ do comparti mento mais facilmente mi neralizável, em relação à ervilhaca soltei ra. No entanto, os resíduos culturais do consórcio entre aveia e ervilhaca apresentaram maior persistência no solo do que aqueles da ervilhaca solteira e liberaram maiores quantidades de $\mathbf{N}$ do que a aveia e o nabo solteiros.
\end{abstract}

Termos de indexação: mineralização de $\mathrm{N}$, taxas de decomposição, composição bioquímica, aveia preta, ervilhaca comum, nabo forrageiro.

\footnotetext{
(1) Parte da Tese de Mestrado do segundo autor, apresentada ao Programa de Pós Graduação em Agronomia, U niversidade Federal de Santa Maria - UFSM. Pesquisa parcialmente financiada com recursos da FAPERGS e do Pronex-CNPq/FINEP. Recebido para publicação em agosto de 2002 e aprovado em maio de 2003.

(2) Professor Adjunto do Departamento de Solos, Universidade Federal de Santa Maria - UFSM. CEP 97105-900 Santa Maria (RS). Bolsista CNPq. E-mail: caita@ccr.ufsm.br

(3) Doutorando do Programa de Pós-Graduação em Ciência do Solo, UFSM. Bolsista CAPES. E-mail: sjgiacomini@mail.ufsm.br
} 


\title{
SUMMARY: CROP RESIDUE DECOMPOSITION AND NITROGEN RELEASE IN SINGLE AND MIXED COVER CROPS
}

\begin{abstract}
Dynamics of decomposition and nitrogen $(\mathrm{N})$ release of cover crop residues in mixed and singl ecultures was eval uated in a field experi ment. Thefol lowing materials wereused: (a) black oat (Avena strigosa Schieb); (b) common vetch (Vicia sativa L.); (c) oil seed radish (Raphanus sativus L. var. oleiferus Metzg.); (d) $32 \%$ black oat $+68 \%$ common vetch, and (e) $51 \%$ black oat $+49 \%$ common vetch. To monitor the residue decomposition, air-dried plant material was inserted in $0.5 \mathrm{~mm}$ mesh nyl on-bags $(0.2$ by $0.2 \mathrm{~m})$, which were placed on the surface of the soil and collected after 15, 29, 43, 59, 71, 82, 112, and 182 days. Remaining dry matter amounts (DM) and $\mathrm{N}$ concentrati ons weredetermi ned in thecoll lected materials. Nonl inear models were fit to the values obtained for the remaining amounts of dry matter and nitrogen, which separated the DM and $\mathrm{N}$ of crop residues in two compartments, one more easily decomposableand the other more recalcitrant. Among the singl ecrops, common vetch presented thehighest decomposition rate $\left(0.06119\right.$ day $\left.^{-1}\right)$ of DM within the more easily decomposable compartment. Where oat was mixed with common vetch there were reductions of $30 \%\left(0.04264\right.$ day $\left.^{-1}\right)$ and $50 \%\left(0.03113\right.$ day $\left.^{-1}\right)$ in the DM decomposition of this compartment when theDM proportion of thegrass was 32 and $51 \%$, respectively. The highest amount of totally rel eased $\mathrm{N}$ occurred during the first 15 days of theexperiment, which lasted 182 days. Results show that an association of oat and common vetch does not reduce the $\mathrm{N}$ rel ease rate of the more easily mineralizable compartment, compared to singlecommon vetch. However, cultural residues of common vetch - oat mixtures presented a higher persistence in the soil than those of singl e common vetch and released higher amounts of $\mathrm{N}$ than single oat and singl e oilseed radish.
\end{abstract}

Index terms: N mineralization, decomposition rates, biochemical composition, black oat, common vetch, oilseed radish.

\section{INTRODUÇÃO}

Nas duas últimas décadas, foram realizados diver sos trabal hos com plantas de cobertura de solo no outono/inverno, tanto no Brasil como em outros países, procurando-se caracterizar a decomposição ea liberação de $\mathrm{N}$ de seus resíduos culturais (Wagger, 1989a; Ranells \& Wagger, 1992; Da Ros, 1993; Ranells \& Wagger, 1996). Esses estudos evidenciaram que as leguminosas, especialmente quando usadas como culturas solteiras, foram rapidamente decompostas após o seu manejo, mesmo em sistema plantio direto, resultando num assincronismo entre a liberação de $\mathrm{N}$ dos seus resíduos culturais e a demanda em $\mathrm{N}$ pelo milho em sucessão. A elevada taxa de decomposição das leguminosas, resultante da baixa relação $\mathrm{C} / \mathrm{N}$ deseus resíduos culturais, também contribui para diminuir a sua eficiência na manutenção da umidade e na proteção do solo contra a erosão (Derpsch et al., 1985).

Consorciando leguminosas e gramíneas, é possível obter uma fitomassa com relação $\mathrm{C} / \mathrm{N}$ intermediária àquela das espécies em culturas solteiras, conforme demonstraram Ranells \& Wagger (1996), para o consórcio entre centeio e ervilhaca peluda, e Heinrichs et al. (2001), para o consórcio entreaveia preta eervilhaca comum. Além da relação $\mathrm{C} / \mathrm{N}$, as proporções dos carboidratos estruturais e lignina também podem ser alteradas nos resíduos culturais de espécies consorciadas (Ranells \& Wagger, 1996). Essas mudanças na composi ção da fitomassa podem provocar al terações na taxa de decomposição dos resíduos culturais, uma vez que esse processo é controlado por diversos atri butos intrínsecos aos resíduos como, por exemplo, as concentrações em fração solúvel em água, em N, em lignina e em polifenóis, além das relações $C / N$, lignina/N elignina + polifenóis/N (Reinertsen et al., 1984; Trinsoutrot et al., 2000).

A liberação de $\mathrm{N}$ dos resíduos culturais do consórcio entre centeio e ervilhaca peluda foi avaliada por Ranells \& Wagger (1996), os quais constataram que a quantidade de $\mathrm{N}$ liberada obedeceu à seguinte ordem: ervilhaca peluda > ervilhaca peluda + centeio >centeio. Nesse mesmo trabalho, o $\mathrm{N}$ liberado dos resíduos culturais do consórcio, nos primeiros 15 dias, foi $13,4 \%$ menor do que aquele encontrado no tratamento com ervilhaca solteira.

Na região Sul do Brasil, ainda são escassas as informações obtidas em condi ções decampo relativas ao estudo da dinâmica de decomposição e de liberação de $\mathrm{N}$ dos resíduos culturais de plantas de cobertura de solo consorciadas no outono/inverno (Heinrichs et al., 2001). O conhecimento desses 
processos é fundamental para que as plantas de cobertura possam ser eficientemente introduzidas no sistema de produção de milho, sendo necessário compatibilizar a máxima persistência dos resíduos culturais na superfície do solo com o fornecimento adequado de $\mathrm{N}$ ao milho.

O presente trabalho teve por objetivo avaliar a decomposiçãoe a liberação de $\mathrm{N}$ deresíduos culturais provenientes de plantas de cobertura de outono/ inverno, cultivadas solteiras e consorciadas.

\section{MATERIAL E MÉTODOS}

\section{Caracterização do local}

O trabalho foi realizado durante o período de outubro de 1999 a março de 2000, na área experimental do Departamento de Solos da Universidade Federal de Santa Maria (UFSM), em Argissolo Vermelho distrófico arênico relevo ondulado de acordo com EMBRAPA (1999) com as seguintes características na camada arável: $150 \mathrm{~g} \mathrm{~kg}^{-1}$ de argila; $\mathrm{pH} \mathrm{H}_{2} \mathrm{O}$ (1:1) 5,5; índice SMP 6,$2 ; 13,4 \mathrm{mg} \mathrm{dm}^{-3}$ de $\mathrm{P} ; 57,5 \mathrm{mg} \mathrm{dm}^{-3}$ de $\mathrm{K}$; $0,0 \mathrm{cmol}_{\mathrm{c}} \mathrm{dm}^{-3}$ de $\mathrm{Al}^{3+} ; 2,5 \mathrm{cmol}_{\mathrm{c}} \mathrm{dm}^{-3}$ de $\mathrm{Ca}^{2+}$; $0,8 \mathrm{cmol}_{\mathrm{C}} \mathrm{dm}^{-3}$ de $\mathrm{Mg}^{2+}$ e $16 \mathrm{~g} \mathrm{dm}^{-3}$ de matéria orgânica.

\section{Delineamento experimental e tratamentos}

O estudo foi realizado com resíduos culturais provenientes de plantas utilizadas para cobertura de solo no outono/inverno, a saber: (a) aveia preta (AP) (Avena strigosa Schieb); (b) ervilhaca comum (EC) (Vicia sativa L.); (c) nabo forrageiro (NF) (Raphanus sativus L. var. oleiferus Metzg.). As plantas de cobertura foram cultivadas no período de maio a setembro de 1999, em sistema sol teiro ou consorciado. No cultivo sol teiro da aveia preta e da ervilhaca comum, foram utilizados $80 \mathrm{~kg} \mathrm{ha}^{-1}$ de sementes, e no cultivo solteiro do nabo forrageiro, $14 \mathrm{~kg} \mathrm{ha}^{-1}$ de sementes, considerando-se um poder germinativo de $100 \%$. Foram utilizados dois sistemas de cultivo consorciado entre aveia e ervilhaca, sendo um com $12 \mathrm{~kg} \mathrm{ha}^{-1}$ de sementes de $\mathrm{AP}+68 \mathrm{~kg} \mathrm{ha}^{-1}$ de sementes de EC e outro com $36 \mathrm{~kg} \mathrm{ha}^{-1}$ de sementes de AP $+44 \mathrm{~kg} \mathrm{ha}^{-1}$ de sementes de EC.

A coleta das plantas para o estudo foi efetuada quando a aveia encontrava-se na fase de pleno florescimento, a ervilhaca no início do florescimento e o nabo forragei ro no final do florescimento. Foram col etadas duas subamostras de $0,36 \mathrm{~m}^{2}$ por parcela de cada tratamento que foram reunidas para compor uma amostra única. Nos sistemas consorciados, procedeu-se à separação da aveia e da ervilhaca para determinar a contribuição de cada espécie na produção total de matéria seca (MS) dos consórcios.
Para facilitar a compreensão dos resultados, os tratamentos serão sempre referenciados em relação à proporção de matéria seca das espécies e não à proporção de sementes utilizadas. Portanto, os tratamentos relativos aos sistemas solteiros e consorciados ficaram assim constituídos: T1 - $100 \%$ AP; T2 - $100 \%$ EC; T3 - $100 \%$ NF ; T4 - $32 \%$ AP + $68 \%$ EC e T5 - $51 \%$ AP + $49 \%$ EC.

O delineamento experimental adotado foi o de blocos ao acaso, com quatro repetições. A unidade experimental consistiu de oito bolsas teladas de nylon com dimensões de $0,2 \times 0,2 \mathrm{~m}$ e mal ha de $0,5 \mathrm{~mm}$ que continham resíduos culturais de cada um dos cinco tratamentos descritos. O total debolsas teladas confeccionadas foi de 160.

A aveia foi separada em colmos, fol has e panículas, a ervilhaca em talos e folhas e o nabo forrageiro em hastes, fol has einflorescências. Estes constituintes, com tamanho de até $19 \mathrm{~cm}$, foram submetidos à secagem ao ar em ambiente protegido ( 25 a $30^{\circ} \mathrm{C}$ ) até massa constante antes de serem colocados nas bolsas, em quantidades equivalentes à produção de cada espécie. A secagem ao ar do material foi feita para evitar possíveis alterações das suas características químicas caso ele fosse submetido à secagem em estufa.

\section{Caracterização química dos resíduos culturais}

Antes da instalação do experimento, foi feita a caracterização química dos resíduos culturais (Quadro 1). Para isso, tomou-se uma amostra de material em cada tratamento para a determinação da quantidade inicial de matéria seca, secando-se o material em estufa a $65^{\circ} \mathrm{C}$ até massa constante. Após a secagem, o material foi pesado e moído em moinho Wiley equipado com peneira de 40 mesh. Nessematerial, foram determi nadas as concentração de C orgânico (C) e N total (N) (Tedesco et al., 1995), celulose (CEL), hemicelulose (HEM) elignina (LIG) (Van Soest, 1963). N os resíduos culturais, também foram feitas determinações da quantidade de matéria seca solúvel em água fria (MSsa), conforme método descrito em Aita (1996), e da sua concentração em C orgânico (Csa) e N total (Nsa) (Tedesco et al., 1995). Para Reinertsen et al. (1984), é importante conhecer esses três atributos nos resíduos culturais para melhor compreender a dinâmica de sua decomposição no solo, principalmente na fase inicial do processo. As quantidades adicionadas de matéria seca, C e N nas bolsas de decomposição com os resíduos culturais de cada tratamento encontram-se no quadro 2.

\section{Avaliações efetuadas}

Em outubro de 1999, as unidades experimentais foram confeccionadas e colocadas na superfície do solo. A avaliação da decomposi ção e da liberação de $\mathrm{N}$ dos resíduos culturais foi feita de acordo com o 
Quadro 1. Características quími cas dos resíduos culturais das plantas de cobertura colocados nas bolsas de decomposi ção

\begin{tabular}{|c|c|c|c|c|c|c|c|c|c|c|c|}
\hline Tratamento(1) & $\mathbf{C}^{(2)}$ & $\mathbf{N}$ & MSsa & Nsa & Csa & CEL & HEM & LIG & $\mathbf{C} / \mathbf{N}$ & Csa/Nsa & LIG/N \\
\hline & \multicolumn{11}{|c|}{ - $\mathrm{g} \mathrm{kg}^{-1}$ de matéria seca } \\
\hline $100 \%$ AP & 443 & 11,1 & 134 & 5,7 & 50,1 & 344,7 & 268,8 & 66,5 & 40,3 & 8,8 & 6,0 \\
\hline $100 \% \mathrm{EC}$ & 432 & 28,8 & 235 & 12,4 & 86,7 & 256,8 & 115,3 & 83,4 & 14,8 & 6,9 & 2,9 \\
\hline $100 \% \mathrm{NF}$ & 416 & 14,2 & 200 & 6,3 & 67,9 & 331,8 & 94,5 & 92,1 & 30,6 & 10,8 & 6,5 \\
\hline $32 \% A P+68 \% E C$ & 448 & 23,9 & 206 & 10,5 & 75,7 & 278,3 & 156,1 & 81,4 & 18,1 & 7,2 & 3,4 \\
\hline $51 \% A P+49 \% E C$ & 425 & 20,9 & 169 & 8,9 & 66,7 & 299,2 & 197,6 & 78,3 & 20,6 & 7,5 & 3,7 \\
\hline
\end{tabular}

(1) AP = aveia preta; EC = ervilhaca comum; NF = nabo forrageiro. ${ }^{(2)} \mathrm{C}=$ carbono; $\mathrm{N}$ = nitrogênio; MSsa = matéria seca solúvel em água; $\mathrm{Nsa}=$ nitrogênio solúvel em água; $\mathrm{Csa}=$ carbono solúvel em água; $\mathrm{CEL}=$ celulose; $\mathrm{HEM}=$ hemicelulose; $\mathrm{LIG}=$ lignina.

Quadro 2. Matéria seca, nitrogênio, carbono, matéria seca solúvel em água, nitrogênio solúvel em água e carbono solúvel em água adicionados nas bolsas de decomposição com os resíduos culturais das plantas de cobertura

\begin{tabular}{|c|c|c|c|c|c|c|c|c|c|c|c|c|}
\hline \multirow{3}{*}{ Tratamento(1) } & \multicolumn{12}{|c|}{ Quantidade adicionada } \\
\hline & \multicolumn{4}{|c|}{ Matéria seca } & \multicolumn{4}{|c|}{ Nitrogênio } & \multirow{2}{*}{ Carbono } & \multirow{2}{*}{ MSsa(2) } & \multirow{2}{*}{ Nsa } & \multirow{2}{*}{ Csa } \\
\hline & AP & EC & $\mathbf{N F}$ & Total & AP & EC & NF & Total & & & & \\
\hline & \multicolumn{12}{|c|}{$-\mathrm{kg} \mathrm{ha}^{-1}$} \\
\hline $\begin{array}{l}100 \% A P \\
100 \% \text { EC } \\
100 \% \text { NF } \\
32 \% A P+68 \% \text { EC } \\
51 \% A P+49 \% \text { EC }\end{array}$ & $\begin{array}{l}4.390 \\
- \\
- \\
1.120 \\
2.160\end{array}$ & $\begin{array}{c}- \\
2.660 \\
- \\
2.370 \\
2.080\end{array}$ & $\begin{array}{l}- \\
- \\
3.720 \\
- \\
-\end{array}$ & $\begin{array}{l}4.390 \\
2.660 \\
3.720 \\
3.490 \\
4.240\end{array}$ & $\begin{array}{c}49 \\
- \\
- \\
17 \\
28\end{array}$ & $\begin{array}{l}- \\
77 \\
- \\
66 \\
60\end{array}$ & $\begin{array}{l}- \\
- \\
53 \\
- \\
-\end{array}$ & $\begin{array}{l}49 \\
77 \\
53 \\
83 \\
88\end{array}$ & $\begin{array}{l}1.950 \\
1.150 \\
1.550 \\
1.560 \\
1.800\end{array}$ & $\begin{array}{l}588 \\
624 \\
744 \\
720 \\
717\end{array}$ & $\begin{array}{l}25 \\
33 \\
23 \\
37 \\
38\end{array}$ & $\begin{array}{l}220 \\
230 \\
252 \\
264 \\
283\end{array}$ \\
\hline
\end{tabular}

(1) AP = aveia preta; $\mathrm{EC}=$ ervilhaca comum; NF = nabo forrageiro. ${ }^{(2)} \mathrm{MSsa}=$ matéria seca solúvel em água; Nsa =nitrogênio solúvel em água; Csa = carbono orgânico solúvel em água.

tempo, realizando-se oi to col etas (uma bolsa/col eta): aos $15,29,43,59,71,82,112$ e 182 dias da instalação das parcelas. Durantea realização do experimento no campo, foram registrados dados de precipitação pluviométrica e temperatura do ar, para subsidiar a interpretação dos resultados (F igura 1).

Após cada período de amostragem, os resíduos vegetais foram submetidos à secagem em estufa a $65^{\circ} \mathrm{C}$ até obtenção de massa constante, para a determinação da matéria seca remanescente (MSR). Nesses materiais, foi tomada uma fração de $1 \mathrm{~g}$, a qual foi incinerada em mufla a $550^{\circ} \mathrm{C}$ durante $4 \mathrm{~h}$ para a determinação de cinzas, expressando-se a matéria seca livre da contaminação com solo, conforme procedimento descrito por Christensen (1985).

Na MSR dos resíduos culturais de cada coleta, foram determinadas as concentrações de $\mathrm{N}$ para calcular as quantidades remanescentes de N (NR). $\mathrm{Na}$ col eta realizada aos 15 dias, também foram determinadas a quantidade de MSsa e a sua concentração em Csa e Nsa.

\section{Análise dos efeitos dos tratamentos}

As taxas de decomposição da matéria seca (MS) e deliberação deN dos resíduos culturais das plantas de cobertura foram estimadas, ajustando-se model os de regressão não lineares aos valores observados, conforme proposto por Wieder \& Lang (1982). Os dois modelos ajustados têm a seguinte equação matemática:

$$
\begin{aligned}
& \text { MSR e NR }=A e^{-k a t}+(100-A) \\
& M S R \text { e NR }=A e^{-k a t}+(100-A) e^{-k b t}
\end{aligned}
$$

em que MSR e NR = percentagem de matéria seca e $\mathrm{N}$ remanescentes no tempo $\mathrm{t}$ (dias); $\mathrm{k}_{\mathrm{a}}, \mathrm{k}_{\mathrm{b}}=$ taxas constantes de decomposição da matéria seca e de liberação de $\mathrm{N}$ do compartimento mais facilmente decomponível (A) e do compartimento mais recal citrante (100-A), respectivamente.

Os dois model os consideram que a MS e o $\mathrm{N}$ dos resíduos culturais podem ser divididos em dois compartimentos. No modelo assintótico (Eq. 1), 


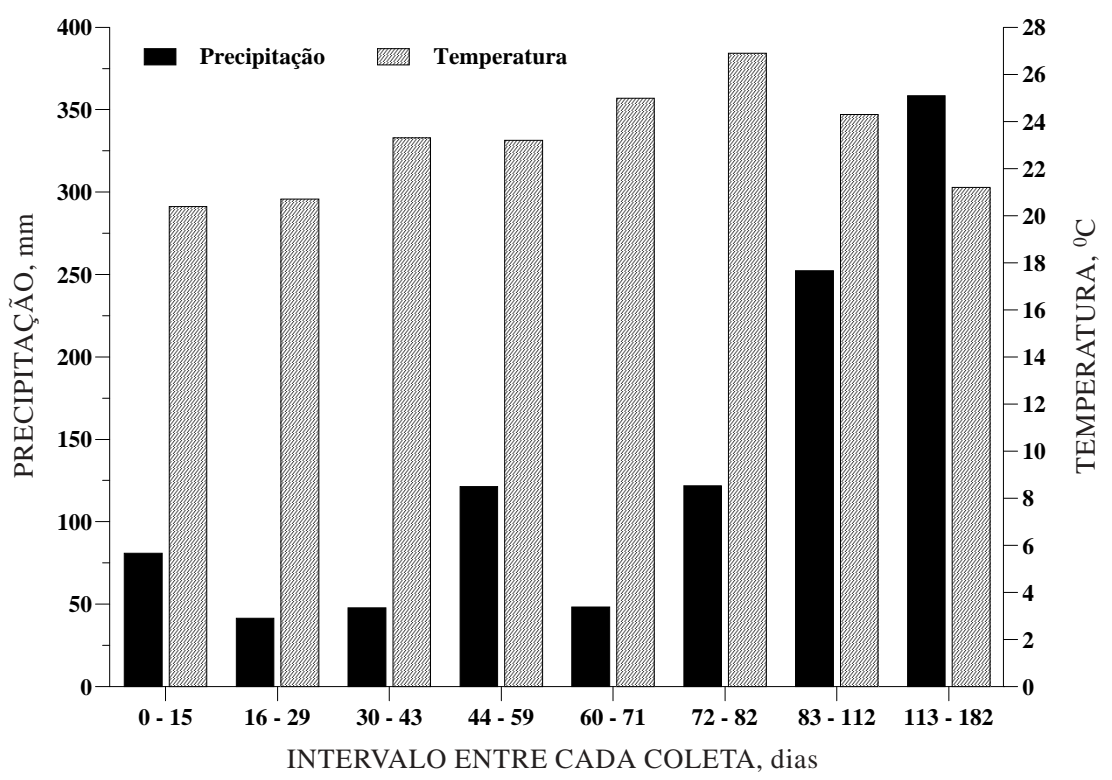

Figura 1. Precipitação pluvial e temperatura média do ar em cada intervalo de coleta dos sacos de decomposição durante o período em que permaneceram no campo.

apenas a MS e o $\mathrm{N}$ do compartimento mais facilmente decomponível (A) são transformados, diminuindo exponencialmente com o tempo a uma taxa constante. A MS eo N dosegundo compartimento (100-A) são considerados mais recal citrantes e, por isso, não sofrem transformação no período detempo considerado. No model o exponencial duplo (Eq. 2), a MS e o $\mathrm{N}$ dos dois compartimentos diminuem exponencialmente a taxas constantes, sendo que a primeira fração é transformada a taxas mais elevadas do que a segunda, que é de mais difícil decomposição (recalcitrante).

A escol ha do tipo de model o em cada tratamento foi feita com base nos valores do coeficiente de determinação $\left(R^{2}\right)$, o qual indica o grau de associação entre o model o ajustado e os valores observados.

A partir dos val ores da constante de decomposição da MS ou da liberação de N de cada compartimento, calculou-se o tempo de meia vida $\left(t_{1 / 2}\right)$, ou seja, o tempo necessário para que $50 \%$ da MS daquele compartimento seja decomposta e $50 \%$ do $\mathrm{N}$ seja liberado. Para este cál culo, utilizou-se a fórmula a seguir, cuja dedução éapresentada em Paul \& Clark (1996):

$$
\mathrm{t}_{1 / 2}=0,693 / \mathrm{k}_{(\mathrm{a}, \mathrm{b})}
$$

Utilizando os model os ajustados para a liberação de $\mathrm{N}$ em cada tratamento, estimou-se a liberação cumulativa de $\mathrm{N}$ durante o período de avaliação. Para isso, multi plicou-se a percentagem deliberação do nutriente em cada col eta, obtida através dos modelos, pelas quantidades inicias de $\mathrm{N}\left(\mathrm{kg} \mathrm{ha}^{-1}\right)$ contidas nos sacos de decomposição (Quadro 2).
Os dados obtidos para a fração solúvel em água fria da primeira coleta e para os parâmetros dos model os ajustados (compartimento $A$, taxas de decomposição eliberação de $N$ et $t_{1 / 2}$ ) foram submetidos à anál ise de variância, comparando-se as médias de tratamento pelo teste Tukey a $5 \%$.

\section{RESULTADOS E DISCUSSÃO}

\section{Decomposição dos resíduos culturais}

Em todos os tratamentos, a cinética do processo de decomposição dos resíduos culturais apresentou um padrão semel hante, com uma fase inicial rápida seguida de outra mais lenta (Figura 2). Ao final do primeiro mês após a colocação das bolsas de decomposição no campo, 81 \% da matéria seca (MS) inicial da aveia ainda permanecia na superfície do solo contra $57 \%$ da ervilhaca, confirmando resultados de outros estudos em que a taxa de decomposição de leguminosas superou a de gramíneas (Ranells \& Wagger, 1992; Da Ros, 1993). O nabo apresentou um comportamento intermediário ao da aveia e ervilhaca sol teiras, uma vez que, nesse período, $75 \%$ da sua MS inicial ainda estava sobre o solo.

A presença da aveia nos consórcios com ervilhaca reduziu a vel ocidade de decomposição dos resíduos culturais em relação à ervilhaca solteira. Com isso, as curvas de decomposição dos dois tratamentos envolvendo consórcio entre aveia e ervilhaca situaram-se num patamar intermediário ao observado nos tratamentos com essas duas espécies 


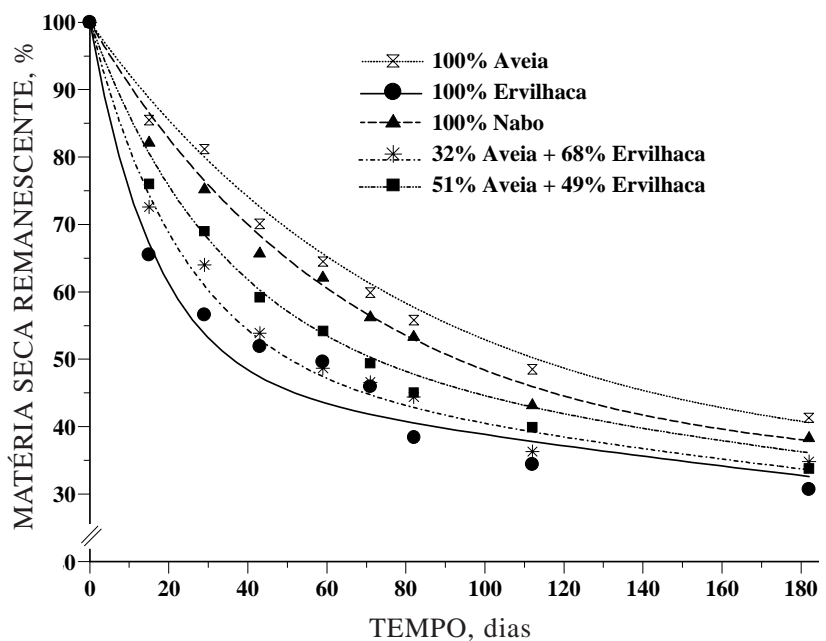

Figura 2. Matéria seca remanescente dos resíduos culturais de plantas de cobertura solteiras e consorciadas, em avaliações realizadas no campo até 182 dias após a distribuição das bolsas de decomposi ção na superfície do solo.

em culturas solteiras (Figura 2). Tais resultados podem ser atribuídos às alterações na composição química da fitomassa dos consórcios em relação às culturas solteiras.

Observa-se, no quadro 1, que as concentrações de $\mathrm{N}$ total (N), N solúvel em água (Nsa) e C solúvel em água (Csa) diminuíram com o aumento na proporção de MS de aveia (AP). Segundo Reinertsen et al. (1984), quanto maior a quantidade de $\mathrm{C}$ e $\mathrm{N}$ solúveis em água, mais rápida será a decomposição inicial dos resíduos culturais, especialmente pela flora bacteriana. Além disso, os compostos carbonados enitrogenados solúveis em água podem ser lixiviados dos resíduos culturais, aumentando sua decomposição inicial (Swift et al., 1979).
O aumento nas relações $\mathrm{C} / \mathrm{N}$ e $\mathrm{Csa} / \mathrm{N}$ sa da fitomassa com o aumento na proporção de MS de aveia (Quadro 1) também deveter contribuído para diminuir a velocidade de decomposição dos resíduos culturais, em relação à ervilhaca solteira. Essa redução na taxa de decomposição, induzida pelo aumento de aveia, constitui-se numa vantagem do consórcio entre aveia e ervilhaca, em relação à ervilhaca solteira, já que ele preserva maior quantidade de resíduos culturais sobre o solo, contribuindo para a manutenção da umi dade do solo e para sua proteção contra o efeito erosivo da chuva.

A análise de correlação linear simples entre a matéria seca remanescente (MSR) em três datas (29, 82 e 182 dias após o manejo das espécies) e alguns constituintes dos resíduos culturais mostrou que a MSR foi inversamente proporcional aos teores de $\mathrm{N}$, Nsa e Csa e diretamente proporcional à relação C/N (Quadro 3), confirmando resultados de outros estudos tanto em condições de laboratório como de campo (Ranells \& Wagger, 1992; Trinsoutrot et al., 2000).

A ervilhaca solteira, com maior concentração de $\mathrm{N}$, Nsa e Csa do que os resíduos culturais da aveia (Quadro 1), apresentou a menor quantidade de MSR (F igura 2). Em trabalho realizado por Trinsoutrot et al. (2000), em condições de laboratório, também houve uma correl ação positiva entre a fração solúvel em água dos resíduos culturais de colza (Brassica napus $L$.) ea sua taxa de decomposição nos primeiros dias após a incubação. Esses autores observaram que, a partir dos 28 dias, outras formas de C, como celulose (CEL) hemicelulose (HEM) elignina (LIG), ea relação LIG/N correlacionaram-se mel hor com a vel ocidade de decomposição do material orgânico.

Para Reinertsen et al. (1984) e Christensen (1985), a el evada taxa inicial de decomposição dos resíduos culturais se deve à facilidade com que os compostos orgânicos, especial mente os carboidratos

Quadro 3. Coeficientes de correlação linear simples entre as quantidades remanescentes de matéria seca e de nitrogênio com algumas características dos resíduos culturais, nas coletas realizadas aos 29, 82 e 182 dias da distribuição das bolsas de decomposição no campo

\begin{tabular}{|c|c|c|c|c|c|c|c|c|c|}
\hline Variável & $\mathbf{N}^{(1)}$ & $\mathbf{C} / \mathbf{N}$ & Nsa & Csa & Csa/Nsa & CEL & HEM & LIG & LIG/N \\
\hline \multicolumn{10}{|l|}{$\mathrm{MSR}^{(2)}$} \\
\hline $\begin{array}{r}29 \text { dias } \\
82 \text { dias } \\
182 \text { dias }\end{array}$ & $\begin{array}{l}-0,994 * * * \\
-0,993 * * * \\
-0,967 *\end{array}$ & $\begin{array}{l}0,951 * * \\
0,951 * * \\
0,967 * *\end{array}$ & $\begin{array}{l}-0,987 * * \\
-0,985 * * \\
-0,942 * *\end{array}$ & $\begin{array}{l}-0,956^{* *} \\
-0,892^{*} \\
-0,902^{*}\end{array}$ & $\begin{array}{l}0,714 \\
0,798 \\
0,704\end{array}$ & $\begin{array}{l}0,992 * * * \\
0,986 * * \\
0,949 * *\end{array}$ & $\begin{array}{l}0,559 \\
0,434 \\
0,518\end{array}$ & $\begin{array}{l}-0,392 \\
-0,277 \\
-0,389\end{array}$ & $\begin{array}{l}0,897 * \\
0,950 * * \\
0,899 *\end{array}$ \\
\hline \multicolumn{10}{|l|}{ NR } \\
\hline $\begin{array}{r}29 \text { dias } \\
82 \text { dias } \\
182 \text { dias }\end{array}$ & $\begin{array}{l}-0,987^{* *} \\
-0,982^{* *} \\
-0,946 * *\end{array}$ & $\begin{array}{l}0,947 * * \\
0,920 * \\
0,979 * *\end{array}$ & $\begin{array}{l}-0,982^{* *} \\
-0,983^{* *} \\
-0,915^{*}\end{array}$ & $\begin{array}{l}-0,853 \\
-0,830 \\
-0,977^{* *}\end{array}$ & $\begin{array}{l}0,852 \\
0,878 \\
0,552\end{array}$ & $\begin{array}{l}0,986 * * * \\
0,994 * * * \\
0,882 *\end{array}$ & $\begin{array}{l}0,433 \\
0,485 \\
0,818\end{array}$ & $\begin{array}{l}-0,297 \\
-0,326 \\
-0,698\end{array}$ & $\begin{array}{l}0,960 * * \\
0,937 * * \\
0,701\end{array}$ \\
\hline
\end{tabular}

(1) N = nitrogênio; Nsa = nitrogênio solúvel em água; Csa = carbono solúvel em água; CEL = celulose; HEM = hemicelulose; LIG = lignina. ${ }^{(2)} \mathrm{AP}=$ aveia preta; EC = ervilhaca comum; NF = nabo forrageiro. ${ }^{(2)} \mathrm{MSR}=$ matéria seca remanescente; NR = nitrogênio remanescente. *,**, ***, significativos a 5,1 e $0,1 \%$, respectivamente. 
da fração solúvel em água, são utilizados como fonte de energia pela população microbiana. Nos primeiros 15 dias após a colocação dos resíduos culturais no campo, houve uma redução, em todos os tratamentos, nas quantidades iniciais de MSsa, Csa e Nsa e na relação Csa/Nsa (Quadro 4). Essa redução foi maior nos valores de MSsa, variando de $68 \%$ na aveia solteira a $86 \%$ na ervilhaca solteira, seguida pelos valores de Csa que variaram de 50 a $78 \%$ nesses mesmos tratamentos.

Considerando que, durante os primeiros 15 dias, a quantidadedeágua das preci pitações pluviométricas ocorridas totalizou 80,9 mm (Figura 1), é provável que, além da decomposição microbiana, parte da diminuição nas quantidades de MSsa, Csa eNsa dos resíduos culturais tenha sido provocada por lixiviação. Todavia, as determinações realizadas não permitem separar a magnitude desses dois processos, os quais ocorrem simultaneamente.

E mbora diversos estudos indiquem que a taxa de decomposição dos resíduos culturais éinversamente proporcional à sua concentração em lignina (Trinsoutrot et al., 2000; Ranells \& Wagger, 1992), isso não foi observado no presente estudo (Quadro 3). Tomando como exemplo as culturas solteiras de aveia eervilhaca, observa-se quea gramínea, apesar de ter apresentado menor proporção de LIG $\left(66,5 \mathrm{~g} \mathrm{~kg}^{-1}\right)$ do quea ervilhaca $\left(83,4 \mathrm{~g} \mathrm{~kg}^{-1}\right)$, foi mais lentamente decomposta do que a leguminosa (Figura 2). Observa-se, noquadro 3, quea proporção entre LIG/N e a concentração de CEL dos resíduos culturais foram os mel hores indicadores da dinâmica da decomposição. Tais resultados evidenciam que, utilizada isol adamente, a concentração de LI G pode não ser um bom indicador da velocidade de decomposição dos resíduos culturais.

Com relação aos modelos avaliados, aquele que melhor se ajustou aos valores de MSR observados na aveia e no nabo sol tei ros foi o assintótico (Eq. 1), enquanto o modelo exponencial duplo (Eq. 2) foi o mais adequado aos valores de MSR dos três tratamentos comervilhaca (Quadro 5). Na ervilhaca solteira, o compartimento mais facilmente decomponível (A) representou $52,3 \%$ da MS dos resíduos culturais cuja taxa constante de decomposição (k) foi de $0,06119 \mathrm{dia}^{-1}$, o que resultou num tempo de meia vida $\left(t_{1 / 2}\right)$ da MS deste compartimento de 11 dias. $\mathrm{Na}$ aveia e no nabo, observa-se que, aproximadamente, $66 \%$ da MS apresentou valores de $\mathrm{k}$ e de meia vida que não diferiram significativamente entre as duas espécies, embora haja uma tendência à maior decomposição dos resíduos culturais do nabo ( $\mathrm{t}_{1 / 2} 11 \%$ inferior ao da aveia).

A alteração na composição bioquímica dos resíduos culturais, pela inclusão da aveia nos consórcios com ervilhaca (Quadro 1), modificou significativamenteal guns parâmetros do modelo, em relação à ervilhaca solteira, especialmente a taxa constante de decomposição $(k)$ do compartimento mais facilmente decomponível. Quando a proporção de MS deaveia foi de $51 \%$, ovalor dek da MS desse compartimento foi praticamente a metade $\left(\mathrm{k}_{\mathrm{a}}=\right.$ $\left.0,03113 \mathrm{dia}^{-1}\right)$ daquele da ervilhaca solteira $\left(\mathrm{k}_{\mathrm{a}}=\right.$ 0,06119 $\mathrm{dia}^{-1}$ ).

A quantidade de MS do compartimento mais facilmente decomponível e os valores da constante de decomposição $\left(k_{b}\right)$ e de meia vida $\left(t_{1 / 2}\right)$ da MS do compartimento mais lentamente decomponível (100A) foram próximos nos três tratamentos com ervilhaca (Quadro 5). Para aproximadamente a metade da quantidade de fitomassa do tratamento comervilhaca solteira e daquele com $51 \%$ AP + $49 \%$ EC, o tempo de meia vida foi de 331 e 341 dias, respectivamente. J á otempo demeia vida dos outros $50 \%$ da fitomassa (compartimento A) desses mesmos tratamentosfoi deapenas 11 e22 dias, respectivamente.

Esse resultado se deve ao fato de que, após a rápida decomposição inicial daquela fração de MS mais facilmente decomponível (Christensen, 1985),

Quadro 4. Matéria seca, carbono e nitrogênio solúveis em água nos resíduos culturais da coleta realizada 15 dias após a distribuição das bolsas de decomposição no campo

\begin{tabular}{|c|c|c|c|c|}
\hline \multirow{2}{*}{ Tratamento(1) } & \multicolumn{4}{|c|}{ Fração solúvel em água } \\
\hline & MSsa(2) & Csa & Nsa & Csa/Nsa \\
\hline & \multicolumn{4}{|c|}{$-\mathrm{kg} \mathrm{ha}^{-1}$} \\
\hline $\begin{array}{l}100 \% \text { AP } \\
100 \% \text { EC } \\
100 \% \text { NF } \\
32 \% \text { AP }+68 \% \text { EC } \\
51 \% \text { AP + } 49 \% \text { EC }\end{array}$ & $\begin{array}{l}187(68)^{(3)} \\
88(86) \\
168(77) \\
131(82) \\
165(77)\end{array}$ & $\begin{array}{r}111(50) \\
51(78) \\
102(60) \\
74(72) \\
107(62)\end{array}$ & $\begin{array}{r}14,7(41) \\
8,9(73) \\
13,5(41) \\
13,4(64) \\
17,5(54)\end{array}$ & $\begin{array}{l}7,6(14) \\
5,7(17) \\
7,5(31) \\
5,5(24) \\
6,2(17)\end{array}$ \\
\hline Tukey $5 \%$ & 96 & 29 & 4,2 & 0,9 \\
\hline
\end{tabular}


Quadro 5. Parâmetros dos modelos ajustados aos valores medidos de matéria seca e nitrogênio remanescente, tempo de meia vida $\left(t_{1 / 2}\right)$ de cada compartimento e valores de $\mathbf{R}^{2}$ em cada tratamento

\begin{tabular}{|c|c|c|c|c|c|c|}
\hline \multirow{2}{*}{ Tratamento(1) } & \multirow{2}{*}{$\mathbf{A}$} & \multirow{2}{*}{$\mathbf{k a}$} & \multirow{2}{*}{$\mathbf{k}_{\mathbf{b}}$} & \multicolumn{2}{|c|}{$\mathbf{t}_{1 / 2}$} & \multirow{2}{*}{$\mathbf{R}^{2}$} \\
\hline & & & & $\mathbf{A}$ & $(100-A)$ & \\
\hline & $\%$ & 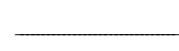 & - & - & - & \\
\hline & \multicolumn{6}{|c|}{ Matéria seca remanescente (MSR) ${ }^{(2)}$} \\
\hline $\begin{array}{l}100 \% \text { AP } \\
100 \% \text { EC } \\
100 \% \text { NF } \\
32 \% A P+68 \% \text { EC } \\
51 \% A P+49 \% \text { EC }\end{array}$ & $\begin{array}{l}66,4 \mathrm{a}^{(4)} \\
52,3 \mathrm{~b} \\
66,3 \mathrm{a} \\
51,1 \mathrm{~b} \\
48,0 \mathrm{~b}\end{array}$ & $\begin{array}{l}0,01359 c \\
0,06119 a \\
0,01507 c \\
0,04264 a b \\
0,03113 b c\end{array}$ & $\begin{array}{l}- \\
0,00209 \\
- \\
0,00207 \\
0,00203\end{array}$ & $\begin{array}{l}51 \mathrm{a} \\
11 \mathrm{~b} \\
46 \mathrm{a} \\
16 \mathrm{~b} \\
22 \mathrm{c}\end{array}$ & $\begin{array}{r}- \\
331 \\
- \\
334 \\
341\end{array}$ & $\begin{array}{l}0,98 \\
0,98 \\
0,99 \\
0,99 \\
0,99\end{array}$ \\
\hline $51 \% A P+49 \% E C$ & \multicolumn{6}{|c|}{ Nitrogênio remanescente (NR)(3) } \\
\hline $\begin{array}{l}100 \% \text { AP } \\
100 \% \text { EC } \\
100 \% \text { NF } \\
32 \% A P+68 \% \text { EC } \\
51 \% A P+49 \% \text { EC }\end{array}$ & $\begin{array}{l}31,4 \mathrm{c} \\
51,9 \mathrm{a} \\
15,3 \mathrm{~d} \\
46,3 \mathrm{ab} \\
39,6 \mathrm{~b}\end{array}$ & $\begin{array}{l}0,04196 \mathrm{~b} \\
0,13313 \mathrm{a} \\
0,18843 \mathrm{a} \\
0,15457 \mathrm{a} \\
0,14420 \mathrm{a}\end{array}$ & $\begin{array}{l}- \\
0,00326 \mathrm{a} \\
0,00286 \mathrm{ab} \\
0,00182 \mathrm{bc} \\
0,00211 \mathrm{bc}\end{array}$ & $\begin{array}{r}16,5 \mathrm{a} \\
5,2 \mathrm{~b} \\
3,7 \mathrm{~b} \\
4,5 \mathrm{~b} \\
4,8 \mathrm{~b}\end{array}$ & $\begin{array}{l}213 \mathrm{a} \\
242 \mathrm{~b} \\
354 \mathrm{~b} \\
328 \mathrm{~b}\end{array}$ & $\begin{array}{l}0,97 \\
0,99 \\
0,97 \\
0,98 \\
0,99\end{array}$ \\
\hline
\end{tabular}

sobram compostos mais recalcitrantes ao ataque microbiano, como, por exemplo, lignina e polifenóis (Paul \& Clark, 1996). Em um estudo realizado em laboratório, Christensen (1985) verificou que, coma remoção da fração solúvel em água dos resíduos da cevada, houve uma redução de 50 \% na evolução de $\mathrm{CO}_{2}$, após 203 dias de incubação a $15^{\circ} \mathrm{C}$.

Analisando a figura 2, observa-se que, seis meses após o manejo das espécies, a percentagem média de MSR dos consórcios entre aveia eervil haca foi de $34 \%$, valor próximo ao obtido com a ervilhaca solteira (31 \%). Todavia, deve-seressaltar queestes percentuais representam apenas $820 \mathrm{~kg} \mathrm{ha}^{-1}$ de MSR na ervilhaca contra $1.320 \mathrm{~kg} \mathrm{ha}^{-1}$ nos consórcios. Essa diferença nas quantidades de MSR, apesar de o percentual de diminuiçãoser próximo, éatribuída à quantidade média de fitomassa adicionada nos consórcios, que foi $46 \%$ superior à da ervilhaca (Quadro 2).

\section{Dinâmica de liberação de $\mathbf{N}$ dos resíduos culturais}

O N remanescente (NR), expresso como percentagem do $\mathrm{N}$ inicial, seguiu o mesmo comportamento observado para a matéria seca remanescente (MSR), apresentando duas fases distintas: uma, nas primeiras duas semanas após o manejo, ondeo $\mathrm{N}$ foi rapidamenteliberado, ea outra, após este período, em que o $\mathrm{N}$ foi liberado mais lentamente (Figura 3).

A quantidade de $\mathrm{N}$ remanescente (NR) foi diretamente proporcional à concentração de celulose (CEL) e às relações $\mathrm{C} / \mathrm{N}$ e LIG/N e inversamente proporcional às concentrações de $\mathrm{N}$ total $(\mathrm{N})$ e $\mathrm{N}$ solúvel em água (Nsa) do tecido vegetal das plantas de cobertura (Quadro 3). No tratamento com ervilhaca solteira, que apresentou a menor relação $\mathrm{C} / \mathrm{N}$ e LIG/N e as maiores concentrações de $\mathrm{N}$ total e Nsa (Quadro 1), foram encontradas as menores quantidades de $\mathrm{N}$ remanescente em toda a fase experimental. Já na primeira amostragem, realizada 15 dias após a distribuição dos sacos de decomposição no campo, apenas a metade do N

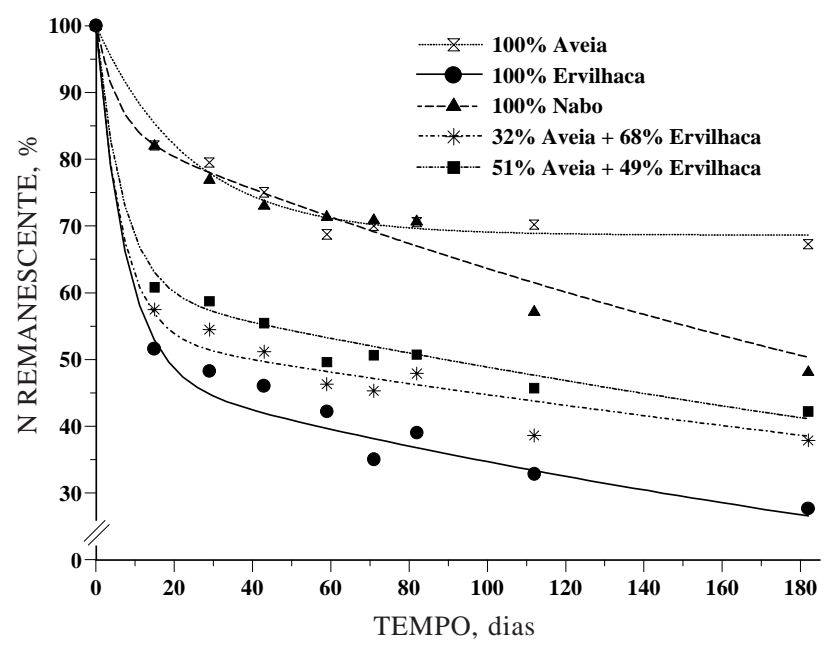

Figura 3. Nitrogênio remanescente dos resíduos culturais de plantas de cobertura solteiras e consorciadas, em avaliações realizadas até 182 dias após a distribuição das bolsas de decomposição na superfície do solo. 
adicionado ainda permanecia nos resíduos culturais (Figura 3). Na aveia, com relação C/N mais el evada $(40,3)$ e menor concentração deNsa (Quadro 1), cerca de $80 \%$ do $\mathrm{N}$ adicionado pela gramínea foi encontrado nos resíduos culturais neste mesmo período. Tais resultados confirmam aqueles obtidos em condições de campo por Wagger (1989b), Amado et al. (2000) e Aita et al. (2001). Em condições controladas, Trinsoutrot et al. (2000) também verificaram que a concentração de $\mathrm{N}$ e a relação $\mathrm{C} / \mathrm{N}$ de 47 tipos de resíduos culturais foram os melhores indicadores da dinâmica do $\mathrm{N}$ no solo.

No nabo, as quantidades de $\mathrm{N}$ remanescentes encontradas nos primeiros 60 dias apresentaram um comportamento semel hante ao observado na aveia solteira (Figura 3). Somente a partir desse período, percebeu-se um comportamento diferenciado entre as duas culturas: oN remanescentenonabo continuou a diminuir e na aveia permaneceu constante (Figura 3). A quantidade de $\mathrm{N}$ remanescente nos resíduos culturais do nabo nos primeiros 30 dias (77\% do $\mathrm{N}$ adicionado) foi elevada, quando comparada àquela encontrada por Basso (1999) de $50 \%$ no mesmo período. A maior quantidade de $\mathrm{N}$ remanescente no nabo, no presente estudo, foi atribuída ao fato de ter sido a cultura col etada após o estádio de florescimento pleno, quando já se observava queda significativa de fol has no campo. Com isso, é provável que tenham aumentado a relação $\mathrm{C} / \mathrm{N}$, a proporção hastes/fol has e a concentração de componentes nitrogenados mais recalcitrantes à decomposição microbiana.

Analisando os dois consórcios entre aveia e ervilhaca, observa-se que, com a inclusão da aveia, foi pequena a redução na vel oci dade de liberação de $\mathrm{N}$, em relação à ervilhaca solteira, sobretudo na fase inicial de decomposição dos resíduos culturais (Figura 3). Esse comportamento pode ser atribuído à composi ção da fitomassa dos consórcios. Observase, no quadro 2, que, mesmo consorciando aveia e ervilhaca, a quantidade de $\mathrm{N}$ acumulada pela leguminosa foi el evada, atingindo, em média, $82 \%$ da quantidade de $\mathrm{N}$ encontrada no tratamento com ervilhaca solteira. Além disso, a relação $\mathrm{C} / \mathrm{N}$ média da matéria seca dos consórcios de 19,4 é mais próxima da ervilhaca $(\mathrm{C} / \mathrm{N}=14,8)$ do que da aveia $(\mathrm{C} / \mathrm{N}=40,3)$.

Considerando as características da aveia, esperava-se que a sua inclusão nos consórcios com a ervilhaca proporcionasse uma redução maior na percentagem de liberação de $\mathrm{N}$ em relação à leguminosa solteira, principalmente na fase inicial da decomposição dos resíduos culturais. Todavia, resultados semel hantes a esses também foram encontrados por Ranells \& Wagger (1996), ao avaliarem a liberação de $\mathrm{N}$ dos resíduos culturais de centeio e ervilhaca peluda. Esses autores concluíram que, quando a matéria seca produzida pelas duas espécies consorciadas foi equivalente, não houve redução significativa na liberação de N, em relação ao monocultivo da leguminosa.

Em conformidade ao observado anteriormente para a evolução das quantidades de MS, o modelo assintótico se ajustou mel hor aos valores observados para a evolução das quantidades de $\mathrm{N}$ na aveia, enquanto, nos tratamentos com ervilhaca, o model o exponencial dupl o foi o mais adequado. O fato de o model o exponencial duploter-seajustado melhor aos valores de NR do nabo do que aos valores de MSR (Quadro 5) indica quea diminuição das quantidades de $\mathrm{N}$ nos resíduos culturais da crucífera foi proporcional mente maior do queaquela ocorrida na MS.

Comparando as culturas sol teiras, observa-se que a taxa constante deliberação do $\mathrm{N}$ do compartimento mais facilmente mineralizável não variou significativamente entre o nabo e a ervilhaca sol teira, apresentando um tempo de meia vida $\left(t_{1 / 2}\right)$ de 3,7 e 5,2 dias, respectivamente (Quadro 5). Todavia, a proporção do $\mathrm{N}$ presente nesse compartimento foi cerca de 3,4 vezes maior na ervilhaca em relação ao nabo (51,9 vs. $15,3 \%$ ). Quantoà aveia solteira, o N contido no compartimento mais facilmente mineralizável situou-se num nível intermediário ao observado na ervilhaca e no nabo, embora a taxa constante de liberação de $\mathrm{N}$ tenha sido significativamente inferior ao dessas duas culturas. Na aveia, o modelo ajustado considerou que $68,6 \%$ do $\mathrm{N}$ dos resíduos culturais foram recalcitrantes, não sendo liberados durante os 182 dias do experimento.

Os val ores dek $\mathrm{k}_{\mathrm{a}}$ não diferiram entreos tratamentos com ervilhaca solteira (EC) e aqueles com os consórcios entre aveia e ervilhaca (Quadro 5). Porém, na ervilhaca, o model o ajustado estimou que $51,9 \%$ do $\mathrm{N}$ dos resíduos culturais da leguminosa fazem parte do compartimento mais facilmente mineral izável contra apenas 39,6 \% no consórcio com $51 \%$ de MS de aveia. Tais resultados indicam que, para quantidades equival entes de $\mathrm{N}$ adicionadas com esses dois tratamentos, haverá maior quantidade de $\mathrm{N}$ liberada dos resíduos culturais da ervilhaca solteira em relação ao consórcio com 51 \% de MS de aveia, apesar de as taxas constantes de decomposi ção serem próximas.

Comparandoa ervilhaca solteira ao consórcio com $32 \% A P+68 \%$ EC, observa-se que todos os parâmetros do modelo para o $\mathrm{N}$ mais facilmente mineralizável desses dois tratamentos não diferiram entre si. Apenas o valor da taxa constante de liberação do $\mathrm{N}$ mais recalcitrante $\left(k_{b}\right)$ diferiu entre esses tratamentos, resultando num tempo de meia vida de 213 dias na ervilhaca solteira e de 354 dias no consórcio $32 \%$ AP + $68 \%$ EC.

U ma das vantagens almejadas com o consórcio entre leguminosas e não-leguminosas consiste em diminuir a velocidade de liberação de N logo após o manejo das plantas de cobertura, em relação às 
leguminosas solteiras, visando otimizar a sincronia entre a liberação de $\mathrm{N}$ dos seus resíduos culturais e a demanda em $\mathrm{N}$ pelas culturas que se deseja instalar em sucessão. Todavia, os resultados deste estudo evidenciam que é pequena a redução na vel ocidade de liberação do $\mathrm{N}$ dos resíduos culturais, quando a ervilhaca é consorciada com aveia.

\section{Estimativa das quantidades de $\mathbf{N}$ li berado dos resíduos culturais}

A maior quantidade de $\mathrm{N}$ liberado ocorreu nos primeiros 15 dias após o manejo (Figura 4), atingindo $36,0 \mathrm{~kg} \mathrm{ha}^{-1}$ de $\mathrm{N}$ na ervil haca solteira, que equivale a $46 \%$ do $\mathrm{N}$ adicionado (Quadro 2 ) e $33,0 \mathrm{~kg} \mathrm{ha}^{-1}$ de N notratamento $51 \% \mathrm{AP}+49 \% \mathrm{EC}$ (38 \% do N adicionado). Comportamentosemelhante foi observado por Amado et al . (2000): a quantidade de $\mathrm{N}$ liberado na ervilhaca solteira nos primeiros 15 dias foi semel hante à do consórcio entre aveia + ervilhaca. Valores relativamente mais distantes entre as quantidades liberadas durante o primeiro mês de decomposição da ervilhaca peluda quando em cultura solteira (92 kg ha-1 de $\mathrm{N}$ ) e quando consorciada ao centeio ( $62 \mathrm{~kg} \mathrm{ha}^{-1}$ de $\mathrm{N}$ ) foram encontrados por Ranells \& Wagger (1996).

A rápida liberação do $\mathrm{N}$ dos resíduos culturais dos tratamentos, incluindo a ervilhaca, poderá contribuir para aumentar as perdas de $\mathrm{N}$, diminuindo o potencial da leguminosa como fonte de $\mathrm{N}$ às culturas comerciais. A elevada taxa de amonificação, especialmente do N solúvel, poderá resultar em perdas de $\mathrm{N}$ por volatilização de amônia, como constataramJ ansen \& McGinn (1991) durante a decomposição dos resíduos culturais de lentilha (Lens culináris Medic.). Considerando que o N amonificado é rapidamente nitrificado, poderão

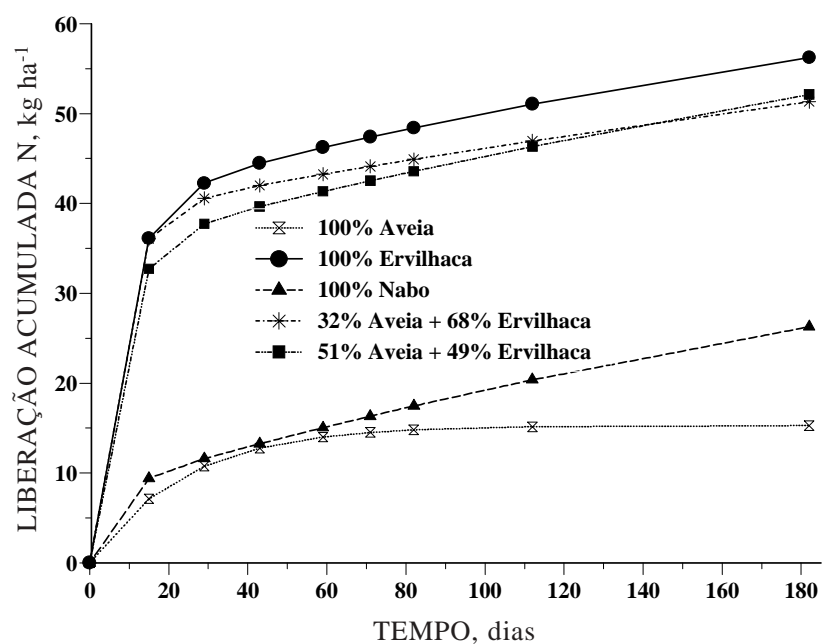

Figura 4. Liberação acumulada de nitrogênio dos resíduos culturais de plantas de cobertura solteiras e consorciadas, em avaliações realizadas até 182 dias após a distribuição das bolsas de decomposição na superfície do solo. ocorrer perdas de $\mathrm{N}$ também por lixiviação de $\mathrm{N}$ $\mathrm{NO}_{3}{ }^{-}$, caso o volume de água das chuvas seja suficiente para ocasionar percolação de água no perfil do solo (McCracken et al., 1994; Giacomini, 2001), e por denitrificação em locais de anaerobiose no solo (Aulakh et al., 1991).

Acredita-se quea magnitude das perdas de $\mathrm{N}$ por esses três processos seja maior na fase inicial de decomposição dos resíduos culturais da ervilhaca, já que, em sistemas de culturas, as espécies comerciais foram recém-implantadas e a sua demanda em $\mathrm{N}$ é praticamente nula. Trabalhando em sistema plantio direto, Giacomini (2001) constatou que o milho após a ervilhaca acumulou apenas $12 \mathrm{~kg} \mathrm{ha}^{-1}$ de $\mathrm{N}$ na parte aérea no primeiro mês após a sua semeadura e que a quantidade de $\mathrm{N}-\mathrm{NO}_{3}{ }^{-}$, na camada de $30-60 \mathrm{~cm}$ do solo do tratamento com ervilhaca, superou aquela do tratamento em pousio invernal em $5,3 \mathrm{~kg} \mathrm{ha}-1$, evidenciando o maior potencial de perdas de $\mathrm{N}$ por lixiviação de $\mathrm{N}-\mathrm{NO}_{3}{ }^{-}$após a leguminosa.

No intervalo entre 29 e 82 dias, quando a demanda em $\mathrm{N}$ pelas culturas comerciais é el evada, as quantidades de $\mathrm{N}$ liberado dos resíduos culturais das plantas de cobertura foram muito pequenas (Figura 4). O valor médio de aproximadamente $7,0 \mathrm{~kg} \mathrm{ha}^{-1}$ de $\mathrm{N}$ dos três tratamentos que continham ervilhaca estão muito aquém das necessidades das culturas para o alcance de tetos el evados de produtividade, especialmente de cereais como o milho.

O N liberado pela ervil haca sol teira 182 dias após o manejo da leguminosa foi de $56 \mathrm{~kg} \mathrm{ha}^{-1}$ de $\mathrm{N}(73 \%$ do $\mathrm{N}$ adicionado) contra apenas $15 \mathrm{~kg} \mathrm{ha}^{-1}$ de $\mathrm{N}$ na aveia (F igura 4). Os dois tratamentos com aveia e ervilhaca consorciadas liberaram, em média, $52 \mathrm{~kg} \mathrm{ha}^{-1}$ de N (61 \% do N adicionado). E mbora a quantidade de $\mathrm{N}$ liberado do tecido vegetal do nabo (27,1 kg ha-1 de $\mathrm{N}$; $51 \%$ do $\mathrm{N}$ adicionado) tenha superado a da aveia, ela foi metade daquela observada na ervilhaca solteira. Estes resultados evidenciam a importância das leguminosas de outono/inverno na mel horia do balanço de N no solo. Todavia, considerando a elevada taxa de liberação do $\mathrm{N}$ das leguminosas logo após o seu manejo, é fundamental que a pesquisa busque estratégias para maximizar o aproveitamento desse $\mathrm{N}$ pelas culturas comercias.

A maior, ou menor, proximidade entre as quantidades de $\mathrm{N}$ liberadas pelas leguminosas solteiras e quando consorciadas com gramíneas dependerá nãoapenas da taxa com que oN éli berado dos resíduos culturais, mas também da quantidade total de $\mathrm{N}$ presente na fitomassa das espécies. No presente estudo, as quantidades de $\mathrm{N}$ liberadas pela ervilhaca e pelos consórcios foram próximas (Figura 4), porque os consórcios adicionaram, em média, $11 \%$ mais $\mathrm{N}$ pela fitomassa do que a ervilhaca (Quadro 2). 
Os resultados relativos à liberação de $\mathrm{N}$ indicam que, provavelmente, nas condições do Sul do Brasil, onde, na primavera, as condições climáticas são favoráveis à decomposição dos resíduos culturais das plantas de cobertura, dificilmente será possível otimizar a sincronia entre a liberação de N com a demanda de $\mathrm{N}$ pelas culturas comerciais. Mesmo consorciando leguminosas e não-leguminosas e em sistema plantio direto, onde os resíduos culturais permanecem na superfície do solo, a liberação de N na fase inicial de decomposição supera a quantidade de $\mathrm{N}$ acumulado pelas culturas comerciais.

\section{CONCLUSÕES}

1. Coma consorciação de aveia eervilhaca, houve uma redução na decomposição dos resíduos culturais, em relação à ervilhaca solteira, porém o efeito dessa prática sobre a vel ocidade de liberação de N na fase inicial de decomposição foi pequena.

2. A velocidade de decomposição e liberação de $\mathrm{N}$ dos resíduos culturais das plantas de cobertura foi inversamente proporcional às relações C/N eLIG/ $\mathrm{N}$ e diretamente proporcional às concentrações de $\mathrm{N}$ total na fitomassa e de $\mathrm{N}$ e $\mathrm{C}$ da fração solúvel em água.

3. Na ervilhaca solteira e nos consórcios de aveia +ervilhaca, a maior parte do $\mathrm{N}$ foi liberada durante os primeiros quinze dias de decomposição dos resíduos culturais.

\section{LITE RATURA CITADA}

AITA, C. Couplage des cycles du carbone et de l'azote dans les sols cultivés: étude, au champ, des processus de decomposition après apport de matière organique fraîche. Paris, UniversitéParis VI, 1996. 196p. (Tesede Doutorado)

AITA, C.; BASSO, C.J .; CERETTA, C.A.; GONÇALVES, C.N. \& DA ROS, C.O. Plantas de cobertura de solo como fontes de nitrogênio ao milho. R. Bras. Ci. Solo, 25:157-165, 2001.

AMADO, T.J.C.; MIELNICZUK, J . \& FERNANDES, S.B.V. Leguminosas eadubação mineral como fontes de nitrogênio para o milho em sistemas de preparo do solo. R. Bras. Ci. Solo, 24:179-189, 2000.

AULAKH, M.S.; DORAN, J.W.; WALTERS, D.T.; MOSIER, A.R. $\&$ FRANCIS, D.D. Nitrate leaching as influenced by cover cropping and nitrogen source. Soil Sci. Soc. Am. J ., 55:10201025, 1991.

BASSO, C.J . Épocas de aplicação de nitrogênio para o milho cultivado em sucessão a plantas de cobertura de solo, no sistema plantio direto. Santa Maria, U niversidade Federal de Santa Maria, 1999. 91p. (Tese de Mestrado)
CHRISTENSEN, B.T. Wheat and barley straw decomposition under field conditions: effect of soil type and plant cover on weight loss, nitrogen and potassium content. Soil Biol. Biochem., 17:691-697, 1985.

DA ROS, C.O. Plantas de inverno para cobertura do solo e fornecimento de nitrogênio ao milho em plantio direto. Santa Maria, Universidade Federal de Santa Maria, 1993. 85p. (Tese de Mestrado)

DERPSCH, R.; SIDIRAS, N. \& HEINZMANN, F.X. Manejo do sol o com coberturas verdes de inverno. Pesq. Agropec. Bras., 20:761-773, 1985.

EMPRESA BRASILEIRA DE PESQUISA AGROPECUÁRIA EMBRAPA. CentroNacional de Pesquisa de Solos. Sistema Brasileiro de Classificação de Solos. Brasília, 1999. 412p.

GIACOMINI, S.J. Consorciação de plantas de cobertura no outono/inverno e fornecimento de nitrogênio ao milho em sistema plantio direto. Santa Maria, Universidade Federal de Santa Maria, 2001. 124p. (Tese de Mestrado)

HEINRICHS, R.; AITA, C.; AMADO, T.J .C. \& FANCELLI, A.L. Cultivo consorciado de aveia e ervilhaca: relação $\mathrm{C} / \mathrm{N}$ da fitomassa e produtividade do milho em sucessão. R. Bras. Ci. Solo, 25:331-340, 2001.

J ANSEN, H.H. \& McGINN, S.M. Volatileloss of nitrogen during decomposition of legumegreen manure. Soil Biol. Biochem., 23:291-297, 1991.

MCCRACKEN, D.V.; SMITH, M.S.; GROVE, J.H.; MacKOWN, C.T. \& BLEVINS, R.L. Nitrate leaching as influenced by cover cropping and nitrogen source. Soil Sci. Soc. Am. J., 58:1476-1483, 1994.

PAUL, E.A. \& CLARK, F.E. Soil microbiology and biochemistry. 2.ed. Califórnia, Academic Press, 1996. 340p.

RANELLS, N.N. \& WAGGER, M.G. Nitrogen release from crimson clover in relation to plant growth stage and composition. Agron. J ., 84:424-430, 1992.

RANELLS, N.N. \& WAGGER, M.G. Nitrogen release grass and legume cover crop monocultures and bicultures. Agron. J ., 88:777-782, 1996.

REINERTSEN, S.A.; ELLIOTT, L.F.; COCHRAN, V.L. \& CAMPBELL, G.S. The role of available $\mathrm{C}$ and $\mathrm{N}$ in determining the rate of wheat straw decomposition. Soil Biol. Biochem., 16:459-464, 1984.

SWIFT, M.J .; HEAL, O.W. \& ANDERSON, J .M. Decomposition in terrestrial ecosystem. Oxford, Blackwell, 1979. 372p.

TEDESCO, M.J .; GIANELLO, C.; BISSANI, C.A.; BOHNEN, H. \& VOLKWEISS, S.J . Análises de solo, plantas e outros materiais. Porto Alegre, Universidade Federal do Rio Grande do Sul, 1995. 174p. (Boletim Técnico, 5)

TRINSOUTROT, I.; RECOUS, S.; BENTZ, B.; LINÈRES, D.; CHÈNEBY, D. \& NICOLARDOT, B. Biochemical quality of crop residues and carbon and nitrogen mineralization kinetics under nonlimiting nitrogen conditions. Soil Sci. Soc. Am. J ., 64:918-926, 2000. 
VAN SOEST, P.J. Use of detergent in the analysis of fibrous feeds I. Preparation of fiber residues of low nitrogen content. J . Assoc. Off. Anal. Chem., 46:825-835, 1963.

WAGGER, M.G. Cover crop management and nitrogen rate in relation to growth and yield of no-till corn. Agron. J ., 81:533538, 1989a.
WAGGER, M.G. Time of desiccation effects on plant composition and subsequent nitrogen release from several winter annual cover crops. Agron. J ., 81:236-241, 1989b.

WIEDER, R.K. \& LANG, G.E. A critique of the analytical methods used in examining decomposition data obtained from litter bags. Ecology, 63:1636-1642, 1982. 